\title{
THE EFFECT OF STREPTOCOCCUS HEMOLYTICUS INFECTION ON THE REACTION OF THE BLOOD OF RABBITS*
}

\author{
LESTER R. DRAGSTEDT
}

From the Pathological Laboratory of Rush Medical College, Chicago

It has been amply demonstrated by many workers using a wide variety of methods of examination that the reaction of the blood remains constant in health and is subject to slight actual variations in disease. Human blood is faintly alkaline and expressed in a physicochemical sense has a hydrogen-ion concentration only slightly less than that of pure water. As determined by its dissociation into hydrogen and hydroxyl ions, pure water at $20 \mathrm{C}$. contains approximately 1-10,$000,000 \mathrm{gm}$. of hydrogen ions to the liter and an equivalent amount of hydroxyl ions; in other words, pure water is $1-10,000,000 \mathrm{~N}$ acid and also $1-10,000,000 \mathrm{~N}$ alkaline. This reaction is conveniently expressed in terms of the hydrogen-ion concentration by the use of logarithmic exponents, such as $10^{7}$ or as suggested by Sörensen ${ }^{1}$ as $P_{H} 7$. When human blood is titrated by electrometric methods its reaction is found to be $P_{H} 7.45$, and the limits of variation to be well within $P_{H} 7.0$ and $P_{H}$ 8.0. The neutral point $P_{H} 7.0$ is reached only in severe acidosis (as a terminal finding) and the limit of alkalinity $\mathrm{P}_{\mathrm{Ir}} 8.0$ only after the prolonged administration of alkalies. This precise regulation of the reaction of the blood constitutes one of the great constants of the body. The acid radicals produced through the oxidation of carbon, sulphur, and phosphorus in the food under normal conditions and in large part the acid radicals which arise through deranged metabolism in disease are either oxidized or neutralized in the body and then excreted or excreted unchanged. The fixed bases in the food and the ammonia arising from protein metabolism furnish bases for such neutralization, and the excretion of carbon dioxid by the lungs and of acid by the kidneys complete the mechanism. In addition the blood itself is able to take care of considerable quantities of either acid or alkali without change in its reaction through the buffer action

Received for publication Aug. 7, 1920.

* This study was made possible by a grant by the Committec on Scientific Research of the American Medical Association.

1 Ergebn. d. Physiol., 1912, 12, p. 393. 
of the carbonates and phosphates of its plasma. From the recent experiments of Bayliss, ${ }^{2}$ it is probable that the proteins of the plasma play no perceptible part in the maintenance of neutrality between the limits of hydrogen-ion concentration possible in the living organism.

While it is thus seen that the reaction of the blood is delicately adjusted by a relatively efficient compensatory mechanism, nevertheless in pathologic conditions under which there may be an excessive production of normal acids as well as abnormal acids or a rapid excretion of bases or a defective excretion of acids, a breakdown in this mechanism occurs. Such a failure in the compensatory mechanism is called acidosis, and the general conception of this condition is that there occurs a diminution in the reserve supply of fixed bases in the blood and other tissties of the body, the physicochemical reaction of the blood remaining unchanged except in extreme conditions. With such a conception it has been definitely demonstrated that an acidosis occurs in diabetes, acute nephritis, ${ }^{3}$ and in certain alimentary disturbances in children. ${ }^{4}$ Many workers have reported acidosis in febrile conditions and in acute infections, ${ }^{5}$ a situation which might be anticipated were there an associated injury to the kidneys. In very few cases of acute infections, however, has an actual increase in the hydrogen-ion concentration of the blood been reported and nome in which an actual acidity, i. e.. al concentration greater than $\mathrm{P}_{\mathrm{H}} 7.0$ was present.

In the recent epidemic of influenza, secondary systemic invasion by Streptococus hemolyticus was frequently. observed and in thesc cases was probably responsible for death. A common necropsy finding was edema of the brain and the so-called cloudy swelling of the parenchymatous organs, changes which have been explained on the basis of Fischer's theories on the action of body colloids as due to an acidosis. It is quite evident that the hydration capacity of the tissue colloids could not be increased through a simple depletion of plasma carbonate with no change in the actual reaction of the blood. since this increased capacity is assumed to be due to the presence of free acid. An acidosis of rather marked grade might therefore be expected in Streptococcus hemolyticus infections.

$\because$ Jour. of Physiol., 1919, 53, p. 162 .

"Sellards, A. H.: Bull. Iohns Hopkins Hosp, 1912, 23, p. 289, and 19!4, 25, y. 141.

* Howlanil, J.. atd Marriott, W. McK., Bull. Johns Hopkins Hosp., 1916, 27, p. 63,

Friderica, L. S., and Olsen, Otto, Deutsch. Arch. f. klin. Med., 19t2, 107. 1. 236.

Walker. I. C.. and Frothingham. C.. Arch. Int. Med.. 1916, 18. p. 304. 
In a recent paper Fleming ${ }^{6}$ has laid great stress on the reaction of the blood and tissue fluids as an important part of the protective mechanism of the body both in resisting and overcoming infection. He states that "The normal reaction of the blood fluids corresponds to an N/35 alkaline solution" and he believes that this degree of alkalinity greatly restricts the growth of such bacteria as B. welchii. Almroth Wright ${ }^{7}$ has demonstrated that $\mathrm{B}$. welchii grows luxuriantly in blood serum which has been "neutralized" by the addition of 2 volumes of $N / 10$ sulphuric acid to 7 volumes of serum, or blood serum which has been "acidified" by adding 3 volumes of $N / 10$ sulphuric acid to 7 volumes of serum but that it does not grow at all in untreated blood serum. He believes that the fact that the avalanche-like invasion of the organism by the gas bacillus after a certain critical point in the infection has been reached may be due in part to the developing acidosis. In a later paper ${ }^{8}$ Wright and Fleming have demonstrated that a similar condition obtains for the other organisms present in cases of gas gangrene, i. e., vibrion septique, Bacillus oedematiens, Bacillus fallax, Bacillus aerofoetidus of Weinberg. Attention should be called to the fact that there is an entire misconception of the chemistry of the blood in the statement that. its normal reaction corresponds to an N/35 alkaline solution. The fact that the blood is equivalent to such solution in its power to neutralize acid must not be interpreted to mean that bacteria would encounter the same resistance to growth in both mediums. N/35 refers to the titratable alkalinity of the blood and not to its actual alkalinity. Furthermore the "neutralized" and "acidified" blood serum in the experiment of Wright and Fleming represent a degree of acidosis that has never been found during life and indeed could not occur.

Other investigators have conceived that the development of acidosis in an acute infection might represent an attempt at resistance in the production of a medium of a reaction unfavorable for bacterial proliferation. As seen from the previous considerations, such a change in the reaction of the blood is difficult of production and can only result after a breakdown in the compensatory mechanism; furthermore, the utmost limits of range of hydrogen-ion concentration possible in the living organism lie between $10^{-4} \mathrm{~N}$ and $10^{-10} \mathrm{~N}$ or $\mathrm{P}_{\mathrm{H}} 4$ and

- Brit. Jour. Surg., 1919, 7, p. 99.

7 Lancet, 1917, 1, p. 1.

${ }^{8}$ Lancet, 1918 1, p. 205. 
$P_{\mathrm{H}} 10^{2}$. Jones ${ }^{9}$ found that a culture of virulent Streptococcus hemolyticus uniformly died out when the reaction of the medium reachel $\mathrm{P}_{\mathrm{H}} 5.11$ or $\mathrm{P}_{\mathrm{H}} 4.63$ when ascitic fluid was added to the medium. Other bacteria, such as B. typhosus, B. paratyphosus, and B. proteus, likewise show a definite limit of hydrogen-ion tolerance.

In order that the reaction of the body fluids might then resist the invading organism through the development of acidity it is necessary to demonstrate that the body fluids actually develop a hydrogenion concentration equal to the limit of the hydrogen-ion tolerance for the organism in question.

Streptococus hemolyticus was chosen for these experiments because its hydrogen-ion tolerance in mediums enriched by body protein is definitely known and also because it seemed possible that it would be quite a powerful agent in causing acidosis, not only through its toxic effect on body cells in general, but also because of its specific hemolyzing effect on the red blood corpuscles. As suggested to me by Dr. Le Count there is definite anatomical evidence for the occurrence of acidosis in streptococcus infections. In comparing the segments of brains cut by serial section after the usual hardening in formol solution, it has been noted for a number of years in this laboratory that with streptococcus infections the swelling and paleness of the brain has been quite like that of the brains of persons dying from uremia and diabetic coma, diseases in which an acidosis develops some time before death. Rabbits were chosen since they are quite susceptible to streptococcus infections and are large enough to withstand the repeated bleedings necessary for blood examinations. The determination of the presence of acidosis involved a consideration only of those methods for measuring an actual increase in the hydrogen-ion concentration of the blood. The usual tests for acidosis, such as increase in tolerance to sodium bicarbonate, lowering of the carbon dioxide of the alveolar air and of the blood, and increase in the output of ammonia in the urine, were discarded since they indicate only a depletion of fixed bases and give no information concerning the actual reaction of the blood. A reduction of the bicarbonate reserve of the blood may be associated with an increase, a decrease, or no change in the hydrogen-ion concentration. An exceedingly accurate measurement of the hydrogen-ion concentration of the blood may be obtained by means of the hydrogen electrode. However, if the hydrogen electrode is to be used for this purpose

Jour. Infect. Dis., 1920, 26, p. 160. 
the gaseous atmosphere with which the blood is brought into equilibrium must contain carbon dioxide at a tension equal to the alveolar carbon dioxide tension of the experimental animal. Such a measurement of the alveolar carbon dioxide tension in experimental animals is very difficult and the method is not practicable. Levy, Rowntree, and Marriott ${ }^{10}$ have introduced a method of measuring the hydrogenion concentration of the blood by the use of indicators, which is simple, readily applicable to animal experiments of this nature, and sufficiently accurate to demonstrate changes that could have any significance for this work. It consists in determining the reaction of the dialysate of oxalated whole blood by the addition of a definite quantity of phenolsulphonephthalein and a comparison of the resulting quality of color with that of colored standard phosphate mixtures of known hydrogen-ion concentration.

REACTION OF TIIE BLOOD IN NORMAL RABBITS

Blood collected from the marginal ear vein of rabbits, oxalated, dialyzed through collodion sacs, and the hydrogen-ion concentration of the dialysate determined according to the method of Levy, Rowntree, and Marriott. Thirty determinations were made on eight normal rabbits at varying intervals during the day with the following results:

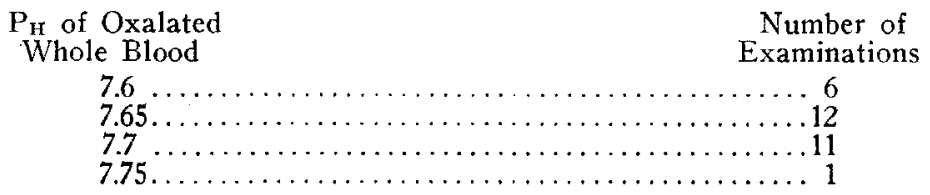

Thus of 30 examinations the reaction of the whole blood was found to range between $P_{H} 7.6$ and 7.75. Levy, Rowntree, and Marriott found the reaction of human blood under normal conditions to vary between $P_{\mathrm{H}} 7.4$ and 7.6. The higher degree of alkalinity in rabbits (herbivora) is probably due to the fact that the diet is rich in free bases and finds confirmation in the normal alkaline reaction of the urine.

\section{REACTION OF THE BLOOD IN STREPTOCOCCUS HEMOLYTICUS INFECTIONS}

Strains of hemolytic streptococci isolated from spinal fluids, pleural fluids, and peritoneal fluids in human infections were used. From 2

10 Arch. Int. Med., 1915, 16, p. 389. 


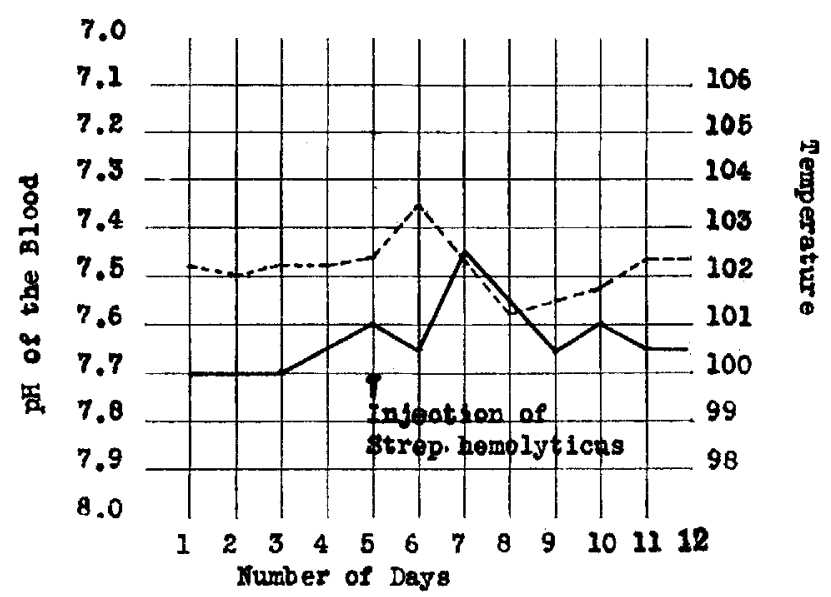

Chart 1 (Exper. 1).-Effect of intraperitoneal injection of $1 \mathrm{c} c$ of a 24-hour culture of St. hemolyticus isolated from pleural fluid. The broken line indicates temperature, the con. tinuous line is the curve of reaction of the blood.

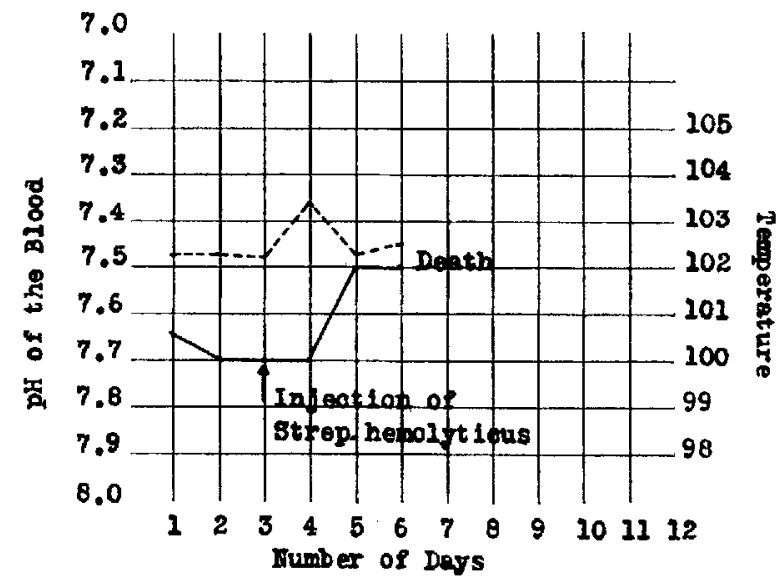

Chart 2 (Exper, 1).-Result of intraperitoneal injection of $2 \mathrm{c} c$ of a 24-bour broth culture of St. hemolyticus isolated from the heart blood of a rabbit dying from a streptococcus infeciion. Death occurred 72 hours later. Necropsy revealed a dry plastic fibrinous general peritonitis. St. hemolyticus was isolated in pure culture from the peritoneal cavity and the heart blood. The broken line indicates temperature; the continuous line is the curve of reaction of the blood. 


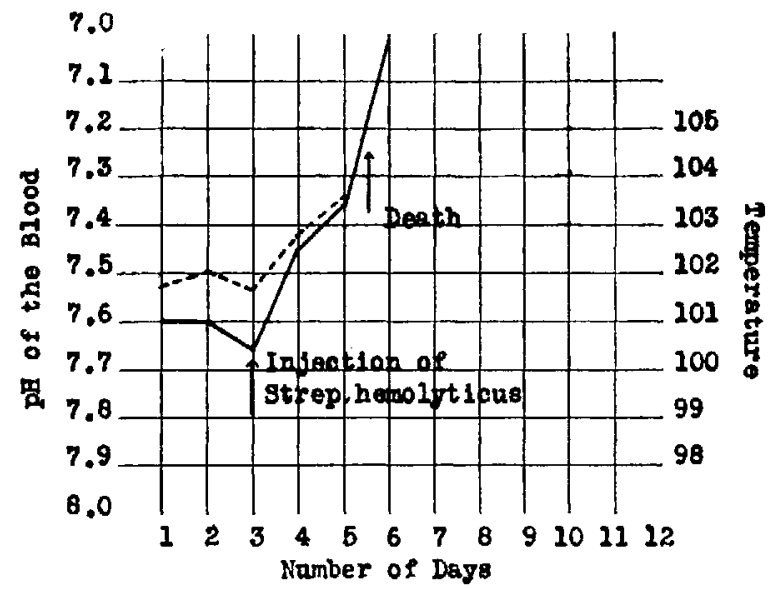

Chart 3 (Exper. 1).-Result of intraperitoneal injection of $2 \mathrm{cc}$ of a 24-hour broth culture of St. hemolyticus isolated from the heart blood of a rabbit dying from a streptococcus infection. Death occurred 48 hours later. Necropsy revealed a general serofibrinous peritonitis. St. hemolyticus was isolated in pure culture from the peritoneal cavity and the heart blood. The marked degree of acidosis found in the blood after deatl may probably be explained by the fact that the excretion of acids by way of the lungs and kidneys ceases immediately at death while the production of acids through the metabolism of cells continues for varying periods thereafter. The broken line indicates temperature; the continuous line is the curve of reaction of the blood.

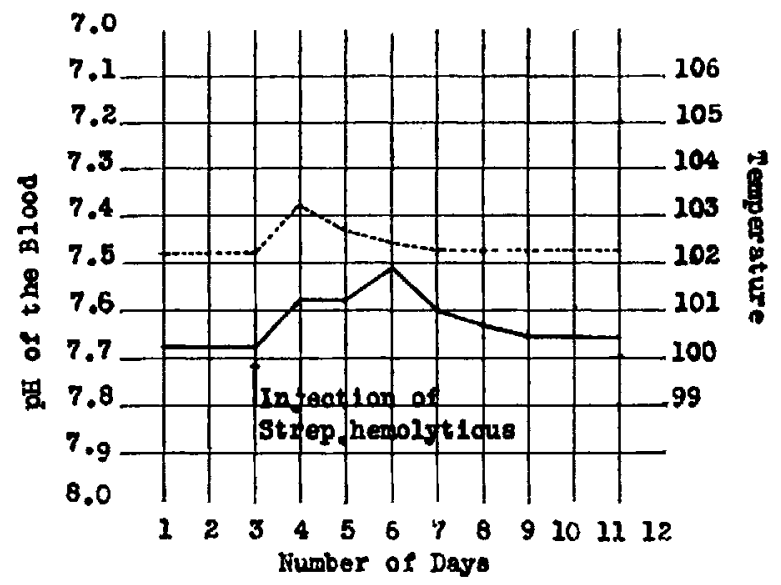

Chart 4 (Exper. 1).-Composite curve showing the effect on the temperature and reaction of the blood of rabbits following the intraperitoneal injection of from 2 to $4 \mathrm{cc}$ of broth cultures of St. hemolyticus (16 experiments). An intraperitoneal injection of killed strepto. cocci in amounts equal to those used for the other experiments produced practically no rise in temperature nor variation in the reaction of the blood greater than occurs under normal conditions with this method of determination. The broken line indicates temperature; the continuous line is the curve of reaction of the blood. 
to $4 \mathrm{cc}$ of a 24 -hour broth culture of these strains was injected intraperitoneally and subsequent daily observations of the gencral condition, temperature, weight, and determinations of the reaction of the blood were made. The following curves are arranged to show the variations in the $P_{\mathrm{H}}$ of the blood during the course of the infections. The animals lost weight following inoculation, but the loss was not marked during the brief period of each experiment so the curves are not plotted.

\section{DISCLSSION}

The maxinum acidity observed during life in any of the animals in the course of the experiments was $P_{H} 7.3$ and even after death the reaction of $P_{\mathrm{H}} 7.0$ indicated an acidity equal to that of pure water. When it is recalled that Streptococcus hemolyticus can grow in medium enriched with serum until the acidity reaches $P_{11} 4.63$ it is evident that the development of acidosis per se in an acute infection can play no inhibitory ròle in the progress of the infection. As a clange in the reaction of the blood toward the alkaline side is even more difficult of attainment due to the ready availability of acids in the body, it is likewise evident that for the reaction of the body fluids to play any part in the mechanism of resistance to bacterial infection the range of hydrogen-ion tolerance and of hydroxyl-ion tolerance of the specific organism in question must lie within the range found in the blood during life, i. e., betwcen $P_{H} 7.0$ and $P_{I I} 8.0$. There remains, of course, the possibility that the alteration in the reaction of the body fluids occurring during the course of the infection may in some way influence the fixation of antigens, the elaboration of specific immune bodies, or the activity of the leukocytes. These are points which are beyond the scope of the present investigation.

\section{CONCLUSIONS}

The blood of normal rabbits is slightly more alkaline than human blood.

Experimental Streptococcus hemolyticus infections in rabbits produce a relatively marked acidosis, the $\mathrm{P}_{\mathrm{H}}$ of the blood varying from 7.65 which is normal, to 7.3 in an extreme case.

The development of acidosis in Streptococcus hemolyticus infections in rabbits does not in itself play any effectual rôle in the mechanism of resistance. 